\title{
Correction of aberration in holographic optical tweezers using a Shack-Hartmann sensor
}

\author{
Carol López-Quesada, Jordi Andilla, and Estela Martín-Badosa* \\ Optical Trapping Lab—Grup de Biofotònica, Departament de Física Aplicada i Òptica, \\ Universitat de Barcelona, Martí i Franquès 1, Barcelona 08028, Spain \\ ${ }^{\star}$ Corresponding author: estela.martinb@ub.edu
}

Received 19 September 2008; revised 30 December 2008; accepted 13 January 2009; posted 15 January 2009 (Doc. ID 101727); published 13 February 2009

\begin{abstract}
Optical aberration due to the nonflatness of spatial light modulators used in holographic optical tweezers significantly deteriorates the quality of the trap and may easily prevent stable trapping of particles. We use a Shack-Hartmann sensor to measure the distorted wavefront at the modulator plane; the conjugate of this wavefront is then added to the holograms written into the display to counteract its own curvature and thus compensate the optical aberration of the system. For a Holoeye LC-R 2500 reflective device, flatness is improved from $0.8 \lambda$ to $\lambda / 16(\lambda=532 \mathrm{~nm})$, leading to a diffraction-limited spot at the focal plane of the microscope objective, which makes stable trapping possible. This process could be fully automated in a closed-loop configuration and would eventually allow other sources of aberration in the optical setup to be corrected for. (C) 2009 Optical Society of America

OCIS codes: $\quad 230.6120,090.1000,350.4855,090.1995$.
\end{abstract}

\section{Introduction}

Optical tweezers are strongly focused laser beams capable of trapping microscopic particles [1]. They have proved to be a very useful tool for exerting and measuring forces on micrometer-sized objects, and they have promising applications in cell and molecular biology [2]. The introduction of holograms displayed on spatial light modulators (SLMs) has greatly improved the design of optical tweezers [3-6]. SLMs spatially modify the laser wavefront before the focusing step, resulting in a completely programmable dynamic intensity pattern over the sample plane. Wavefront control easily permits three-dimensional positioning of the traps as well as the creation of beams with special characteristics, such as Bessel or Laguerre-Gaussian beams [7], which carry angular momentum.

In optical tweezers, the gradient component of the light force needs to be greater than the scattering component to guarantee stable trapping, and thus high numerical aperture (NA) microscope objectives

0003-6935/09/061084-07\$15.00/0

(C) 2009 Optical Society of America are used to bring light to a tight focus. These are very well corrected to form diffraction-limited optical traps, commonly at small distances from the cover glass. When using oil-immersion objectives to trap samples suspended in water, the quality of the trap degrades rapidly as depth increases due to spherical aberration arising from refractive index mismatch at the glass-water interface $[8,9]$. In $[10,11]$ the axial trapping force was enhance $\overline{\mathrm{b}} \overline{\mathrm{b}}$ correcting this aberration with a deformable mirror. Other aberrations, such as optical misalignment or even aberration caused by the refractive index distribution of the specimen, should be compensated for as well to ensure a diffraction-limited spot. Optical vortices are especially sensitive to aberrations both in their shape and in the distribution of light around their circumference [12-14].

SLMs have proved to be a useful tool for generating Zernike polynomials [15] and correcting aberrations in adaptive optical systems [16-18]. Recently, liquid crystal on silicon (LCoS) technology has provided high resolution, small pixel size, and large fill factors at relatively low costs [19]. An LCoS microdisplay essentially consists of a liquid crystal layer 
sandwiched between a cover glass and a silicon backplane that contains the drive electronics and that is commonly coated with aluminum to ensure high reflectivity. The microdisplay operates by reflection in a double-pass beam path that increases the phase modulation range and thus makes it particularly suitable for wavefront applications in which $2 \pi$ modulation depth is desirable.

Unfortunately, the silicon backplane manufactured using standard CMOS methods is not flat [20], which frequently gives rise to important optical aberration. Such distortion is too strong to ignore when using SLMs for wavefront control in applications such as laser beam steering, diffractive optical element generation, or emulation of atmospheric turbulence. Therefore much effort has been made to measure and correct the static aberrations of LCoS displays, mainly by interferometry. In [18,21-23] a Boulder Nonlinear Systems (BNS) SLM [24] of the XY Phase Series (P256 or P512 model, depending on the reference) is characterized, while in $[25,26]$ a Hana Microdisplay device [27] is analyzed. Furthermore, Holoeye devices [28] are dealt with in [22] (Holoeye HEO 1080P) and [14] (Holoeye LC-R $720)$. In the latter, Jesacher et al. used the high sensitivity of Laguerre-Gaussian modes to aberrations to determine the phase errors from the distorted shape of a focused doughnut mode: a phase retrieval algorithm is applied to find the hologram that would produce the observed distorted doughnut if displayed on an ideally flat SLM and imaged with "perfect" optics. This method could be applied in holographic optical tweezers, in which aberrations of the SLM may dominate and prevent stable trapping of microscopic samples.

Reicherter and co-workers presented [29] a method to correct aberrations in microscopy inspired by astronomy, in which an illuminated microsphere serves as a nearly spherical reference wavefront. Holographic optical tweezers are used to move this artificial point source within the object space, thus enabling spatially selective aberration measurement. The emitted wavefront is analyzed using either a fiber interferometer or a Shack-Hartmann (SH) wavefront sensor. The complex conjugate of the measured distorted wavefront is then written into the same SLM that is used for manipulation of the particle and therefore corrects the trapping light beam itself. This method has been tested for defocus but has never been applied to actual holographic setups. In contrast, Wulff et al. reported [30] a method for correcting aberrations in holographic optical tweezers and showed some results of using a Holoeye LC-R 2500 SLM. The basic idea is to add an appropriate hologram to the SLM display, by arbitrary generation of Zernike polynomials characterizing the unknown wavefront distortion, until a tightly focused spot is obtained, thus optimizing trap performance. The goodness of the correction is quantified by establishing the mean-square displacement of the trapped beads from their respective trapping centers, which is related to trap stiffness. For the SLM used in their study, Wulff et al. showed that astigmatism correction leads to an improvement in the fidelity of the focused spot. The effect of this correction on the performance of the optical trap is most noticeable for small particles $(0.8$ and $2 \mu \mathrm{m}$ silica microspheres). They also state that any quantifiable effect was difficult to separate from other aberrations such as coma, trefoil, and spherical aberration.

We have previously shown [31] that the beam reflected by our Holoeye LC-R $2 \overline{500}$ modulator, placed at $45^{\circ}$ with respect to the optical axis, when focused by the microscope objective in a holographic optical tweezers setup, forms two lines of light instead of a diffraction-limited spot. This prevents stable trapping of microsized particles and imperatively needs to be solved. We manually found a phase function that reversed this effect (similar to astigmatism) and eventually corrected the aberration. However, this was a hard trial-and-error task that needed to be repeated each time the setup was rebuilt to account for slight misalignments of the optical system. Besides, only the central part of the modulator could be corrected for and used for trapping experiments.

We use a SH wavefront sensor to measure the distorted wavefront at the SLM plane. The conjugate of this wavefront is then added to the holograms displayed on the SLM to counteract its own curvature and compensate the optical aberrations of the system. We analyze the quality of the trap before and after correction, and demonstrate that stable trapping is possible after correction using the whole active area of the modulator for hologram generation. We then focus our attention on studying SLM aberration and analyzing whether compensation of loworder astigmatism alone would produce acceptable results. Future work would include exploiting the potentialities of wavefront detection and hologram correction working in a closed-loop configuration in a holographic optical tweezers system, such as dynamic compensation of any source of aberration.

\section{Experimental Setup and Procedure}

Figure 1 shows the layout that combines holographic optical tweezers with adaptive optics for aberration correction. A continuous wave, $\mathrm{TEM}_{00}$ laser beam $(\lambda=532 \mathrm{~nm})$ is first expanded and then collimated by lenses LE and LC. A pinhole spatially filters the light at the back focal plane of the expander lens to ensure clean, Gaussian illumination of the SLM. We used an LCoS reflective SLM (Holoeye LC-R 2500), which has an active area of $19.5 \mathrm{~mm} \times 14.6 \mathrm{~mm}$ and supports digital video interface (DVI) signals with a resolution of $1024 \times 768$ pixels (pixel pitch $19 \mu \mathrm{m}$, fill factor 93\%) [28]. The SLM was tilted $45^{\circ}$ with respect to the optical axis [31] and sandwiched between two linear polarizers (P1 and P2) with proper orientations to provide phase-mostly modulation $[32,33]$. A beam splitter divides the beam in two: one half enters a commercial microscope to 


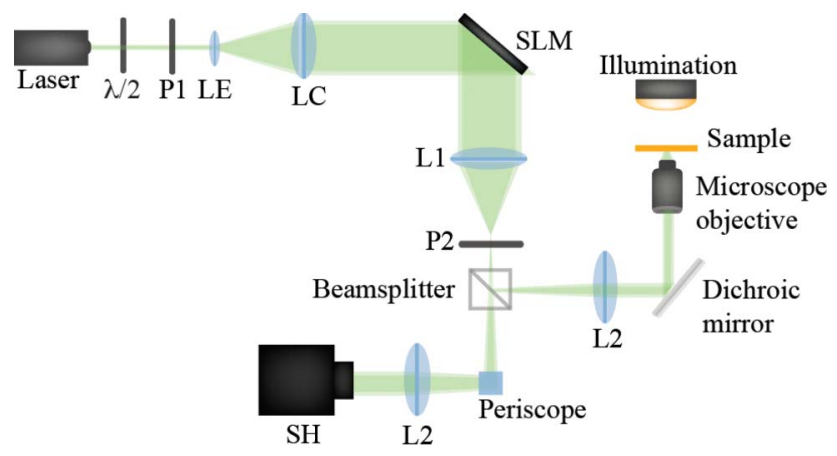

Fig. 1. (Color online) Experimental setup for holographic optical trapping and correction of SLM optical aberrations: LE, expander lens; LC, collimating lens; P1 and P2, polarizers.

trap microscopic particles; the other is redirected to a $\mathrm{SH}$ wavefront sensor. In our setup, a Motic AE-31 inverse microscope equipped with an oil-immersion objective (Motic Planachromat, 100×, 1.25 NA) was used; the beam was reflected upward by a dichroic mirror and focused at the sample plane by the objective. Along the other arm, wavefront distortions were measured with a HASO 32 Imagine Optics sensor [34], which has $32 \times 32$ microlenses and an aperture of $5 \mathrm{~mm} \times 5 \mathrm{~mm}$. The telescope formed by lenses L1 and L2 provided parallel illumination to the infinity-corrected microscope objective and imaged the SLM onto both the exit pupil of the objective and the wavefront sensor. To make use of its whole active area, the image of the modulator was scaled down to match the size of the objective back aperture (around $3.5 \mathrm{~mm}$ in diameter), and at the same time it was fitted into the sensor aperture. Considering that the longest side of the modulator was reduced by a factor of $\sqrt{ } 2$ due to its $45^{\circ}$ tilt, a telescope magnification of 0.25 gave an image of the modulator that slightly overfilled the objective pupil and occupied $22 \times 24$ subapertures of the $\mathrm{SH}$ sensor.

If the SLM is placed at the front focal plane of lens $\mathrm{L} 1$, its image is formed at the back focal plane of lens L2. To keep the overall length of the optical system reasonably small, the focal length of lens L2 is $f_{\mathrm{L} 2}=$ $100 \mathrm{~mm}$, and, consequently, $f_{\mathrm{L} 1}=400 \mathrm{~mm}$. Unfortunately, $100 \mathrm{~mm}$ is too short a distance to allow us to divide the two beams after passing through lens L2 and before entering the microscope: a periscope system is required to adapt the heights of the beams to the microscope and the sensor, which is assembled on a rotating stage with micrometric controls to facilitate its alignment. This means that, even if lens L2 is duplicated in both arms of the system and thus contributes to the final wavefront on the sensor, the latter does not actually measure possible misalignments of lens L2 and the objective lens in the optical tweezers path. However, we are mostly concerned here with the predominant aberrations caused by the modulator, and we ignore distortions derived from incorrect alignment of the system or from the highly corrected microscope objective. Further developments would allow us to measure distortions of the trap itself, which would then enable us to correct other aberrations such as the spherical aberration of oil-immersion objectives trapping deep into an aqueous medium.

To ensure that the main aberration was indeed due to SLM nonflatness, we carefully aligned the optical system together with the $\mathrm{SH}$ sensor, which was set up to receive an incoming wavefront with minimum tilt and defocus. The wavefront aberration was then measured, and a conjugated correction phase (modulo $2 \pi$ ) was displayed on the SLM, taking into account its modulation curve [32,33]. This relates phase modulation with the gray level displayed; as images on the sensor have only $22 \times 24$ pixels, gray level values of the remaining pixels of the SLM were computed by interpolation. Next, the residual wavefront error was measured and added to the previous correction; this procedure was repeated iteratively to obtain a minimal aberration, such that, if no other hologram were added to the SLM, a plane wavefront should be achieved.

\section{Results and Discussion}

The distorted wavefront received by the $\mathrm{SH}$ sensor at the SLM plane is shown in Fig. 2(a); it has a peak-tovalley (PV) value of $7.6 \lambda$ and a root-mean-square (RMS) value of $1.4 \lambda$. These large distortion values are common for reflective LCoS SLMs (see, for comparison, $[18,22,26,30])$ and reveal the lack of flatness of the device, which needs to be corrected to obtain diffraction-limited spots and stable trapping. By applying the procedure described in Section 2, after nine iterations we obtained the correction hologram shown in Fig. 3 , which should give an exit plane

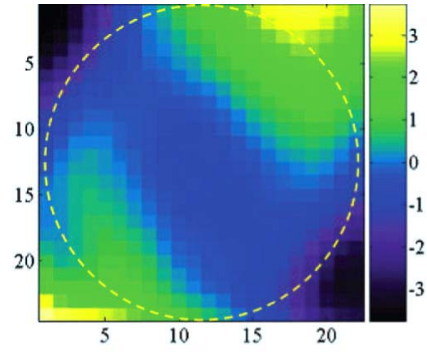

(a)

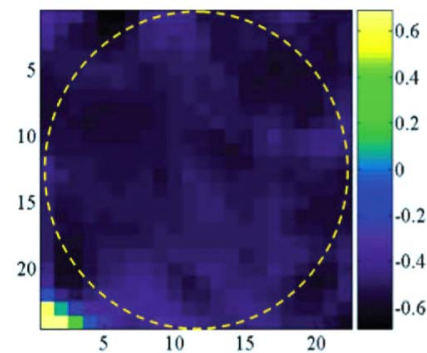

(c)

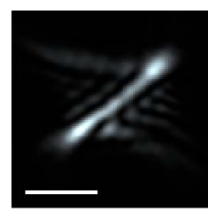

(b)

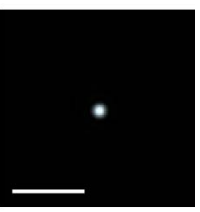

(d)
Fig. 2. (Color online) Wavefront at the SLM plane and the corresponding simulated spot focused by the objective lens (scale bar $2 \mu \mathrm{m}$ ): (a) and (b) before correction; (c) and (d) after correction. 


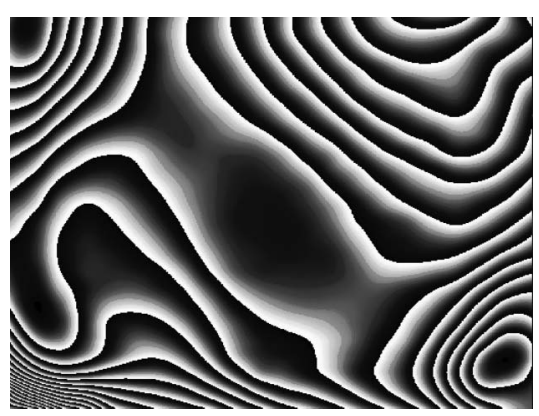

Fig. 3. Hologram displayed on the SLM for self-aberration correction.

wavefront when displayed on the SLM. The residual aberration measured by the sensor is shown in Fig. 2(c), for which the PV and RMS deviations are $1.4 \lambda$ and $0.1 \lambda$, respectively. In the holographic optical tweezers setup, only the inscribed circular area highlighted in Figs. 2(a) and 2(c) enters the microscope objective to avoid diffraction through the rectangular active area of the SLM and to maximize the effective numerical aperture. Then, if we disregard aberrations outside the inscribed round pupil, flatness is improved from $0.8 \lambda$ RMS (4.7 $\lambda$ PV) to $\lambda / 16$ RMS $(\lambda / 3 \mathrm{PV})$, yielding a diffraction-limited spot when focused through the objective lens (the commonly accepted diffraction-limited criterion is a RMS error below $\lambda / 14)$. We believe that residual aberrations close to the edges are due mainly to the spatial nonuniformity of the SLM, meaning that its response actually changes from pixel to pixel; when scaling the conjugated phase into gray-level values, we used a single phase modulation curve, which was measured in a central area of the SLM of about $3 \mathrm{~mm}$ in diameter [33], far away from the edges. As pointed out by other authors [18,22], aberration correction could be improved by using a spatially varying phase response. In fact, our experimental system could be used to determine the local phase modulation in each of the pixels of the wavefront sensor (which corresponds to an SLM area of about $32 \times 32$ pixels) by measuring phase changes when each of the 256 gray-level values is displayed on the modulator. A closed-loop configuration would then be desirable to fully automate the process. Moreover, this would allow iterative correction of SLM distortions without previous knowledge of its phase response, just by trial and error.

Figures 2(b) and 2(d) show the simulated spot at the focal plane of the objective lens before and after correction, respectively. They were computed as the intensity of the Fourier transform of the corresponding wavefront at the SLM plane. This relationship is systematically used in holographic optical tweezers for hologram calculation: Fourier transform applies even for high numerical aperture lenses as long as they are well corrected (Abbe's sine condition is fulfilled) [35]. The scale bar was computed taking into account the real characteristics of our experimental setup (numerical aperture, focal length, and wavelength). Gaussian illumination was also included in the simulation.

We compared the quality of the experimental trap before and after correction: the left column of Fig. 4 shows several images of an uncorrected trap for different planes along the optical axis, revealing the lack of symmetry typical of astigmatism (as we see below when analyzing the aberrations of the system); the circular symmetry of the corrected trap at different planes (right column of Fig. 4) shows the improvement of trap quality after aberration correction. It also proves that the main aberration was indeed due to the SLM and not to misalignment of the optical setup. To quantify the correction goodness, we studied the changes in lateral trap stiffness with the number of iterations. When no correction was applied or after the first iterations, we were not able to trap any particle, and thus we could not measure

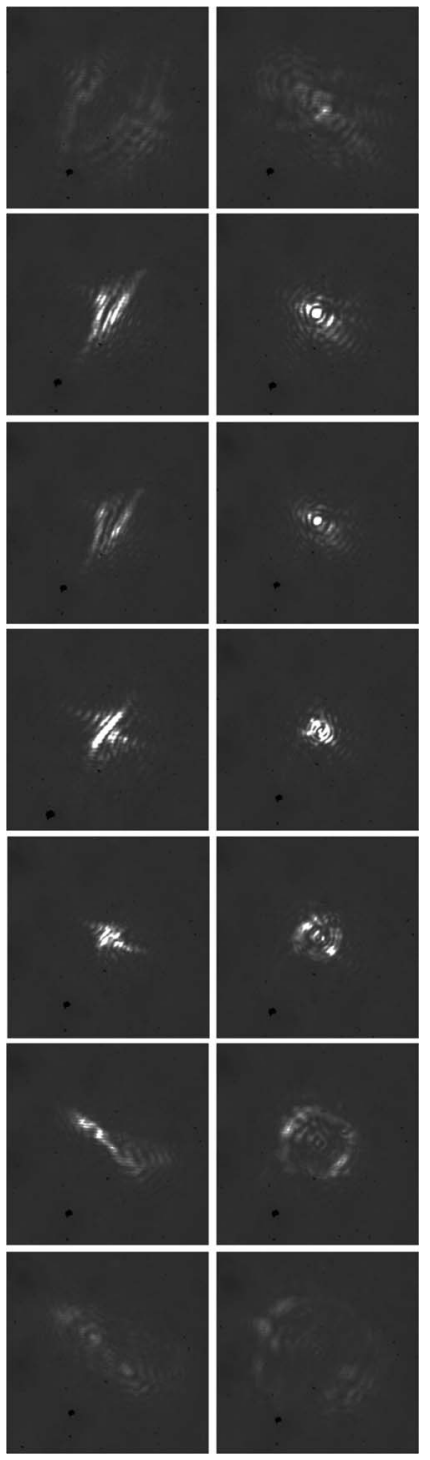

Fig. 4. Images of the experimental trap for different planes along the optical axis, before correction (left column) and after aberration correction (right column). The distance between the top and the bottom plane is around $1 \mu \mathrm{m}$. 


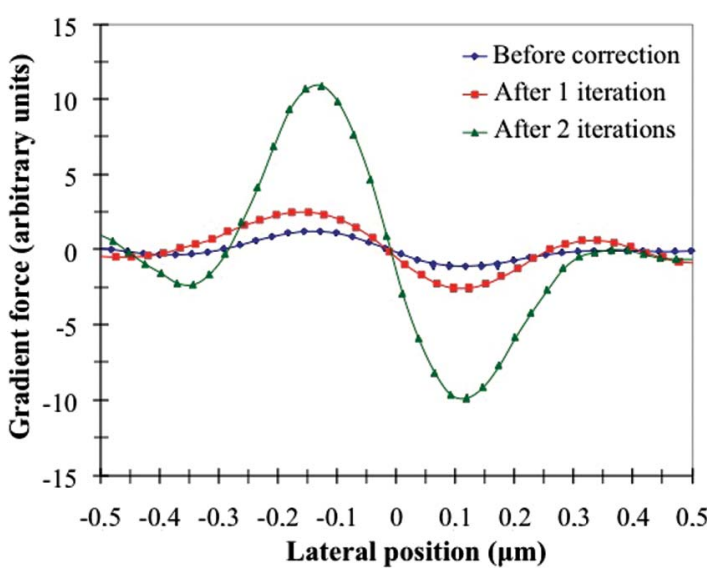

Fig. 5. (Color online) Gradient force before aberration correction and after the first two iterations: the trap stiffness is the slope of the linear region and increases with iteration.

the corresponding spring constant experimentally. Still, we could estimate its value from the slope of the gradient of the simulated spot at the objective focal plane. This is valid in the Rayleigh regime using normalized arbitrary units [36]: we disregard the characteristics (polarizability) of the sample, which is supposed to be the same throughout all the experiments or iterations; the beam power is held constant as well.

We computed the maximum trap stiffness after each iteration. Figure 5 shows a plot of the gradient force for the uncorrected trap and for the corrected traps after one and two iterations, to illustrate the improvement in trap stiffness (slope of the linear region); the three curves are superimposed for comparison (the directions of maximum slope were different). Table 1 lists the lateral trap stiffness in all cases. We can see that its value after nine iterations has improved by a factor of around 34 compared to the original uncorrected trap (iteration 0). Actually, there is a quick increase in trap stiffness after three or four iterations, followed by a slow further improvement, until an optimized trap is obtained, very close to the ideal case corresponding to a perfect focused spot with no aberration. No changes were observed for more iterations. We also checked that there is a correlation between quality of aberration correction (in terms of wavefront RMS errors) and trap stiffness. This agrees with the results presented in [30], in which they correlate spot sharpness and trap performance.

Finally, we used the corrected trap to stably trap and move a $5 \mu \mathrm{m}$ polystyrene bead, as shown in Fig. $\underline{6}$. Linear phase patterns were added to the correction hologram of Fig. 3 to displace the trapped particle to the desired position; spherical wavefronts were also used to move the sample along the optical axis.

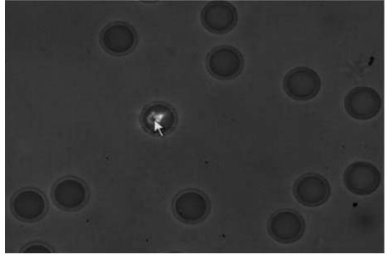

(a)

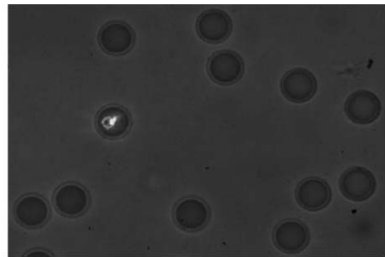

(b)
Fig. 6. (a) Corrected holographic trap moved the $5 \mu \mathrm{m}$ polystyrene bead to the left. (b) The laser light was filtered to avoid saturating the images; the empty circle indicates the trap center.

Note that it was not necessary in our setup to block the central undiffracted beam, which nearly vanished [33].

On the other hand, we further analyzed aberrations of our optical system by decomposing the wavefronts inside the circular pupil in Figs. 2(a) and 2(c) by means of Zernike polynomials. We used the first 32 coefficients, as defined in [37]. Table 2 lists the most significant terms, revealing for the distorted wavefront a predominant astigmatism aberration (both third and fifth orders) followed by third-order spherical aberration. After correction, all the Zernike coefficients were below $\lambda / 20$. Wang et al. reported [26] the predominance of low-order defocus and astigmatism for a Hana Microdisplay device. Hart et al. compared [23] the static aberrations of three BNS devices: they all show important defocus and astigmatism, among other aberrations. The same conclusion can be drawn from [18].

In our case, the system was aligned to obtain minimum tilt and defocus, which are deliberately not shown in Table 2. This means that the beam onto the sensor is collimated, and we actually correct any defocus introduced by the SLM by axially displacing lens L2. This procedure is correct as long as lens L2 in the beam entering the microscope is also displaced; in fact, we believe this could even be helpful when aligning the optical tweezers, as we are forcing the beam to be parallel before it enters the microscope objective. In any case, defocus could be compensated at the end by adjusting the observation and the trapping plane, if necessary. Furthermore, excluding defocus from aberration compensation means measuring a less distorted wavefront with the $\mathrm{SH}$ sensor and displaying a smoother hologram on the SLM, which is desirable.

So far we have seen that aberrations produced by the SLM curvature can be corrected to obtain a diffraction-limited pattern. Ignoring focus effects, an analysis of Zernike coefficients reveals aberration predominantly due to astigmatism, as reported for other LCoS devices. We wonder now whether correction of solely low-order astigmatism would

Table 1. Lateral Trap Stiffness After Each Iteration (Arbitrary Normalized Units), from 0 (Before Correction) to 9 (Ideal Case Corresponds to a "Perfect" Spot with No Aberration)

\begin{tabular}{|c|c|c|c|c|c|c|c|c|c|c|c|}
\hline Number of iterations & 0 & 1 & 2 & 3 & 4 & 5 & 6 & 7 & 8 & 9 & Ideal \\
\hline Lateral trap stiffness & 1.0 & 2.1 & 9.4 & 21.5 & 28.9 & 29.3 & 30.6 & 31.9 & 32.1 & 33.9 & 35.1 \\
\hline
\end{tabular}


Table 2. Zernike Coefficients of Uncorrected and Corrected Wavefronts

\begin{tabular}{lcc}
\hline & \multicolumn{2}{c}{$\begin{array}{c}\text { Zernike Coefficient } \\
\text { (Waves at 532 nm) }\end{array}$} \\
\cline { 2 - 3 } Zernike Term-Aberration & $\begin{array}{c}\text { Before } \\
\text { Correction }\end{array}$ & $\begin{array}{c}\text { After } \\
\text { Correction }\end{array}$ \\
\hline 4-Astigmatism at $0^{\circ}$ & 0.40 & -0.04 \\
5-Astigmatism at $45^{\circ}$ & 1.66 & -0.04 \\
6-Coma at $0^{\circ}$ & 0.04 & 0.00 \\
7-Coma at $45^{\circ}$ & 0.00 & -0.02 \\
8-3rd order spherical & -0.42 & 0.00 \\
9-Trefoil at $0^{\circ}$ & -0.11 & 0.05 \\
10-Trefoil at 45 & 0.14 & -0.05 \\
11-5th order astigmatism at $0^{\circ}$ & 0.48 & 0.00 \\
12-5th order astigmatism at $45^{\circ}$ & 0.19 & 0.00 \\
\hline
\end{tabular}

yield acceptable results; this would allow the use of a simplified method in which no wavefront sensor or interferometric setup would be required, and correction could be achieved by adjusting the few parameters that model low-order astigmatism, as we reported in [31]. When considering the whole circular aperture of the SLM (as indicated in Fig. 2), the RMS deviation after astigmatism correction is $0.4 \lambda(1.6 \lambda$ $\mathrm{PV}$ ), even in the ideal case in which there was no residual tilt or defocus. We studied the quality of astigmatism correction for smaller apertures: Fig. 7(a) shows the evolution of the RMS error of corrected wavefronts as the diameter of the SLM aperture, which is normalized to the maximum inscribed pupil, diminishes. We can see that to achieve the diffraction-limited criterion of $\lambda / 14$ for the RMS value,

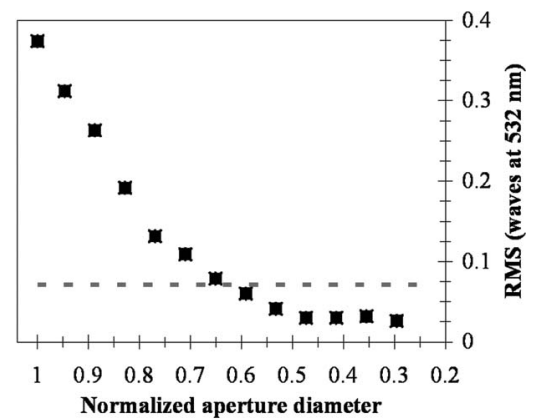

(a)

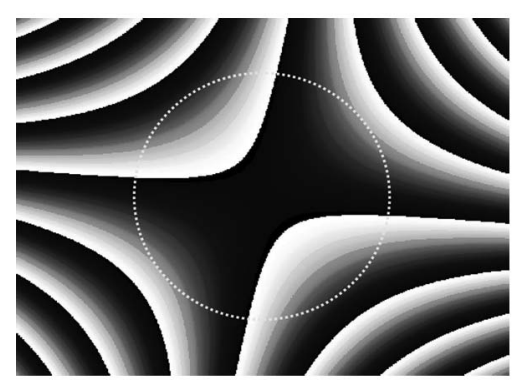

(b)

Fig. 7. (a) Residual RMS error after low-order astigmatism correction, as a function of the normalized SLM aperture; diffractionlimited results are obtained for RMS values below the dashed line. (b) Hologram to compensate astigmatism for an aperture indicated by the dashed line. indicated by a dashed line in Fig. 7(a), we need to reduce the aperture to around 0.65 times the maximum. The hologram displayed on the SLM for this purpose is shown in Fig. 7(b). This means that the nonflatness of our modulator could eventually be compensated this way if a diaphragm were used to ensure that only the central pixels of the device are illuminated, covering around $40 \%$ of its maximum circular aperture or $20 \%$ of the rectangular SLM active area. It also explains our previous work [31]: in that case, the hologram compensated both astigmatism and defocus (lens L2 was not artificially displaced, as it was here) and thus required a larger amount of wave variation. Despite these results, using a $\mathrm{SH}$ sensor is, in our case, a more convenient solution for accurate nonflatness correction without sacrificing spatial resolution, which additionally enables us to compensate other sources of aberration. Still, for other devices it is worth trying low-order astigmatism correction first: for example, good trap performance after correction has been demonstrated in $[30]$.

\section{Conclusion}

We corrected the aberration of an LCoS SLM used in a holographic optical tweezers setup. We measured the distorted wavefront at the SLM plane with a Shack-Hartmann sensor and iteratively computed a compensation phase hologram, which is displayed on the SLM for self-corrections. RMS deviations improved from $0.8 \lambda$ to $\lambda / 16$, ensuring a diffractionlimited trap in the focal plane of the microscope objective, which allows stable trapping and manipulation of microscopic samples. Lateral trap stiffness increases quickly after a few iterations and reaches a plateau of around 34 times the initial value for the uncorrected trap, close to the ideal result for a "perfect" trap. An analysis of Zernike polynomials describing the aberrated wavefront indicates a predominance of astigmatism and third-order spherical aberration. We also demonstrated that correction of low-order astigmatism alone can yield diffraction-limited beams if the active area of the modulator is limited to a central circular aperture. A generalization of this conclusion to other LCoS devices would indicate that aberration can be corrected to some extent with no need for interferometric setups or wavefront sensors. In our case, the use of a $\mathrm{SH}$ sensor provides accuracy and reproducibility and enables a series of enhancements to be considered in the future, especially if the wavefront detection and SLM addressing work in a closed-loop feedback configuration: the process can then be fully automated and dynamic distortion correction is feasible, even with no previous knowledge of SLM phase response, which could actually be characterized this way. Furthermore, additional phase patterns can be considered to correct for other aberrations, such as misalignment. In optical tweezers, apart from the well-known spherical aberration of the microscope objective due to refractive index mismatch, aberrations derived 
from the use of a wavelength of light for which the objective is not corrected and distortions due to nonuniformity of biological samples could also be compensated for. This dynamic generation of wellcorrected traps is one of the benefits of using SLMs in optical tweezers, compared to other beam steering techniques such as acousto-optic deflectors. A prior and necessary step consists in solving the deficiencies of the modulator to guarantee stable holographic trapping, as presented in this paper.

We acknowledge M. Montes-Usategui for many fruitful discussions, E. Pleguezuelos and M. Roth for their help with the alignment of the optical system, F. Gómez-Morales who contributed to the set up of the Shack-Hartmann sensor, and A. Farré for his advice on trap stiffness calculation. This work was funded by the Spanish Ministry of Education and Science under grants FIS2007-65880 and NAN200409348-C04-03.

\section{References}

1. A. Ashkin, J. M. Dziedzic, J. E. Bjorkholm, and S. Chu, "Observation of a single-beam gradient force optical trap for dielectric particles," Opt. Lett. 11, 288-290 (1986),

2. M. J. Lang and S. M. Block, "Resource letter: LBOT-1: Laserbased optical tweezers," Am. J. Phys. 71, 201-215 (2003).

3. M. Reicherter, T. Haist, E. U. Wagemann, and H. J. Tiziani, "Optical particle trapping with computer-generated holograms written on a liquid-crystal display," Opt. Lett. 24, 608-610 (1999).

4. J. Liesener, M. Reicherter, T. Haist, and H. J. Tiziani, "Multifunctional optical tweezers using computer-generated holograms," Opt. Commun. 185, 77-82 (2000).

5. E. R. Dufresne, G. C. Spalding, M. T. Dearing, S. A. Sheets, and D. G. Grier, "Computer-generated holographic optical tweezer arrays," Rev. Sci. Instrum. 72, 1810-1816 (2001).

6. J. E. Curtis, B. A. Koss, and D. G. Grier, "Dynamic holographic optical tweezers," Opt. Commun. 207, 169-175 (2002).

7. D. G. Grier, "A revolution in optical manipulation," Nature 424, 810-816 (2003).

8. O. Muller, M. Schliwa, and H. Felgner, "Calibration of light forces in optical tweezers," Appl. Opt. 34, 977-982 (1995).

9. A. Rohrbach and E. H. K. Stelzer, "Trapping forces, force constants, and potential depths for dielectric spheres in the presence of spherical aberrations," Appl. Opt. 41, 2494-2507 (2002).

10. T. Ota, T. Sugiura, S. Kawata, M. J. Booth, M. A. A. Neil, R. Juskaitis, and T. Wilson, "Enhancement of laser trapping force by spherical aberration correction using a deformable mirror," Jpn. J. Appl. Phys. 42, L701-L703 (2003).

11. E. Theofanidou, L. Wilson, W. J. Hossack, and J. Arlt, "Spherical aberration correction for optical tweezers," Opt. Commun. 236, 145-150 (2004).

12. K. Ladavac and D. Grier, "Microoptomechanical pumps assembled and driven by holographic optical vortex arrays," Opt. Express 12, 1144-1149 (2004).

13. Y. Roichman, A. Waldron, E. Gardel, and D. G. Grier, "Optical traps with geometric aberrations," Appl. Opt. 45, 3425-3429 (2006).

14. A. Jesacher, A. Schwaighofer, S. Fürhapter, C. Maurer, S. Bernet, and M. Ritsch-Marte, "Wavefront correction of spatial light modulators using an optical vortex image," Opt. Express 15, 5801-5808 (2007).
15. G. D. Love, "Wave-front correction and production of Zernike modes with a liquid-crystal spatial light modulator," Appl. Opt. 36, 1517-1520 (1997).

16. J. Liesener, M. Reicherter, and H. J. Tiziani, "Determination and compensation of aberrations using SLMs," Opt. Commun. 233, 161-166 (2004).

17. Q. Mu, Z. Cao, L. Hu, D. Li, and L. Xuan, "An adaptive optics imaging system based on a high-resolution liquid crystal on silicon device," Opt. Express 14, 8013-8018 (2006).

18. J. D. Schmidt, M. E. Goda, and B. D. Duncan, "Aberration production using a high-resolution liquid-crystal spatial light modulator," Appl. Opt. 46, 2423-2433 (2007).

19. S. T. Wu and D. K. Yang, Reflective Liquid Crystal Displays (Wiley, 2001).

20. K. Seunarine, D. W. Calton, I. Underwood, J. T. M. Stevenson, A. M. Gundlach, and M. Begbie, "Techniques to improve the flatness of reflective micro-optical arrays," Sens. Actuators A 78, 18-27 (1999).

21. J. Harriman, A. Linnenberger, and S. Serati, "Improving spatial light modulator performance through phase compensation," Proc. SPIE 5553, 58-67 (2004).

22. J. Otón, P. Ambs, M. S. Millán, and E. Pérez-Cabré, "Multipoint phase calibration for improved compensation of inherent wavefront distortion in parallel aligned liquid crystal on silicon displays," Appl. Opt. 46, 5667-5679 (2007).

23. N. Hart, M. C. Roggemann, A. Sergeyev, and T. J. Schulz, "Characterizing static aberrations in liquid crystal spatial light modulators using phase retrieval," Opt. Eng. 46, 086601 (2007).

24. http://www.bnonlinear.com/

25. X. Wang, B. Wang, J. Pouch, F. Miranda, J. E. Anderson, and P. J. Bos, "Performance evaluation of a liquid crystal-onsilicon spatial light modulator," Opt. Eng. 43, 2769-2774 (2004).

26. X. Wang, B. Wang, P. J. Bos, J. E. Anderson, J. J. Pouch, and F. A. Miranda, "Finite-difference time-domain simulation of a liquid-crystal optical phased array," J. Opt. Soc. Am. A 22, 346-354 (2005).

27. http://www.hanaoh.com/.

28. http://www.holoeye.com/.

29. M. Reicherter, W. Gorski, T. Haist, and W. Osten, "Dynamic correction of aberrations in microscopic imaging systems using an artificial point source," Proc. SPIE 5462, 68-78 (2004).

30. K. D. Wulff, D. G. Cole, R. L. Clark, R. Di Leonardo, J. Leach, J. Cooper, G. Gibson, and M. J. Padgett, "Aberration correction in holographic optical tweezers," Opt. Express 14, 4169-4174 (2006).

31. E. Martín-Badosa, M. Montes-Usategui, A. Carnicer, J. Andilla, E. Pleguezuelos, and I. Juvells, "Design strategies for optimizing holographic optical tweezers setups," J. Opt. A 9, S267-S277 (2007).

32. E. Martín-Badosa, A. Carnicer, I. Juvells, and S. Vallmitjana, "Complex modulation characterization of liquid crystal devices by interferometric data correlation," Meas. Sci. Technol. 8, 764-772 (1997).

33. J. Andilla, S. Vallmitjana, and E. Martín-Badosa, "Prediction of phase-mostly modulation for holographic optical tweezers," Opt. Commun. 281, 3786-3791 (2008).

34. http://www.imagine-optic.com/.

35. K. von Bieren, "Lens design for optical Fourier transform systems," Appl. Opt. 10, 2739-2742 (1971).

36. Y. Harada and T. Asakura, "Radiation forces on a dielectric sphere in the Rayleigh scattering regime," Opt. Commun. 124, 529-541 (1996).

37. R. J. Noll, "Zernike polynomials and atmospheric turbulence," J. Opt. Soc. Am. 66, 207-211 (1976). 